\title{
STEENROD REPRESENTABILITY OF STABLE HOMOLOGY
}

\author{
PETER S. LANDWEBER
}

1. Introduction. N. Steenrod posed the problem of determining those homology classes of a space which can be represented as the image of the fundamental class of a manifold. The problem, which is central in cobordism theory, has since been treated by $\mathrm{R}$. Thom [12], P. Conner and E. Floyd [6], [7], and Y. Shikata [11], among others. In this paper the manifolds will be weakly almost complex (น-manifolds). A solution will be obtained in the stable range for the Eilenberg-MacLane spaces $K(\pi, q), \pi$ a cyclic group of prime or infinite order, and for the spaces $B U[2 q]$ fibred over $B U$ with $\pi_{i}(B U[2 q])=0$ when $i<2 q$ and $\pi_{i}(B U[2 q]) \rightarrow \pi_{i}(B U)$ an isomorphism when $i \geqq 2 q$. The spaces $B U[2 q]$ were studied by J. F. Adams [1], who denoted them $B U(2 q, \cdots, \infty)$.

We now introduce some notation. All spaces will have base points, and all homology and cohomology theories will be reduced. For $p$ a prime, $\rho_{p}: \widetilde{H}_{*}(X ; Z) \rightarrow \widetilde{H}_{*}\left(X ; Z_{p}\right)$ denotes the usual coefficient homomorphism. $\tilde{\mathcal{U}}_{*}(X)=\tilde{H}_{*}(X ; M U)$ is the (graded) complex bordism group of $X$, i.e., the homology of $X$ with coefficients in the Milnor spectrum $M U[7],[10]$. There is a natural transformation $\mu: \tilde{\mathcal{U}}_{*}(\quad)$ $\rightarrow \widetilde{H}_{*}(; Z)$ of homology theories; we denote by $\mu_{p}: \tilde{U}_{*}(X)$ $\rightarrow \tilde{H}_{*}\left(X ; Z_{p}\right)$ the composition $\rho_{p} \mu$. In terms of $\mu$, the problem posed above becomes that of determining the image of $\mu$ in $\tilde{H}_{*}(X ; Z)$; see [6] for the geometric interpretation of $\mu$.

The main results, along with several of an auxiliary nature, are summarized below. Their proofs are given in $\$ \$ 3$ and 4 . Some observations on the homology of a spectrum, with coefficients in another spectrum, are collected in $\$ 2$.

Lemma (1.1). For any of the spaces $K(\pi, q), \pi$ cyclic of prime or infinite order, or $B U[2 q], \cap_{p}$ ker $\left(\rho_{p}\right)=0$ in the stable range.

TheOREM (1.2). For any of the spaces $K(\pi, q), \pi$ cyclic of prime or infinite order or $B U[2 q], a$ homology class $x$ in the stable range is in the image of $\mu$ if and only if $\rho_{p}(x)$ is in the image of $\mu_{p}$ for all primes $p$.

We are therefore led to consider the homomorphisms $\mu_{p}: \tilde{\mathcal{U}}_{*}(X)$ $\rightarrow \widetilde{H}_{*}\left(X ; Z_{p}\right)$. Let $A^{*}(p)$ denote the $\bmod p$ Steenrod algebra, and let $\chi: A^{*}(p) \rightarrow A^{*}(p)$ denote the canonical anti-automorphism [9]. Given $a \in A^{i}(p)$, make $a$ act on $\widetilde{H}_{*}\left(X ; Z_{p}\right)$ by means of the Kronecker index:

Received by the editors July 4, 1966. 


$$
\langle a x, \xi\rangle=\langle x, \chi(a) \xi\rangle
$$

for $x \in \tilde{H}_{k}\left(X ; Z_{p}\right)$ and $\xi \in \tilde{H}^{k-i}\left(X ; Z_{p}\right)$; thus $a$ lowers degrees by $i$ in homology. Let $I^{*}(p)$ denote the two-sided ideal in $A^{*}(p)$ generated by the Bockstein $Q_{0}^{(p)}$.

Lemma (1.3). For any space with base point, the image of $\mu_{p}$ is annihilated by all (homology) operations in $I^{*}(p)$.

Theorem (1.4). For $X$ any of the spaces $K(\pi, q), \Pi$ cyclic of prime or infinite order, or $B U[2 q]$, the image of $\mu_{p}$ in the stable range is exactly the subspace of $\tilde{H}_{*}\left(X ; Z_{p}\right)$ annihlated by $I^{*}(p)$.

The following result may be of independent interest. Adams has defined classes $c h_{q, r} \in H^{2 q+2 r}(B U[2 q] ; Z)$ for $q \geqq 0$ and $r \geqq 0$.

Proposition (1.5). For $r<q$, one can construct $y_{q, r} \in \tilde{u}_{2 q+2 r}(B U[2 q])$ so that $\left\langle\mu\left(y_{q, r}\right), c h_{q, r}\right\rangle=1$.

2. Homology of a spectrum. The families of spaces $K(\pi, q)$ and $B U[2 q]$, as well as the Thom spaces $M U(q)$, all yield spectra. We denote these spectra by $K(\pi), b u$ and $M U$. (The spectrum $b u$ has spaces $b u_{2 q}=B U[2 q], b u_{2 q-1}=\Omega B U[2 q] ; M U$ has spaces $M U_{2 q}=M U(q), M U_{2 q+1}=S^{1} \wedge M U(q)$.) If $\boldsymbol{M}=\left[M_{q}\right]$ denotes one of these spectra, then $\boldsymbol{M}$ has the following properties:

(a) $M_{q}$ is $(q-1)$-connected;

(b) $S M_{q} \rightarrow M_{q+1}$ induces isomorphisms $\pi_{i}\left(S M_{q}\right) \rightarrow \pi_{i}\left(M_{q+1}\right)$ for $i<2 q$ and an epimorphism for $i=2 q$.

We want to be able to prove results of the following sorts: $\tilde{\mathcal{U}}_{2 q+2 r}(B U[2 q])$ is free abelian for $r<q ; \tilde{\mathcal{U}}_{2 q+2 r+1}(B U[2 q])=0$ if $r<q-1$; the map $M U(q) \rightarrow B U[2 q]$ obtained from the $K$-theory Thom class induces an epimorphism $\tilde{\mathrm{U}}_{2 q+2 r}(M U(q)) \rightarrow \tilde{\mathrm{U}}_{2 q+2 r}(B U[2 q])$ for $r<q$. Such results may be proved by letting $q \rightarrow \infty$ to obtain homology groups of a spectrum with coefficients in another spectrum - e.g., $H_{r}(b u ; M U)$-and by then noticing that we may interchange the roles of the two spectra and re-examine the questions. E.g., $H_{r}(b u ; M U) \approx H_{r}(M U ; b u)$, and the latter group may be investigated with the help of the homology theory $\widetilde{H}_{*}(; b u)$. These ideas are due to A. Hattori [8].

Let $\boldsymbol{M}=\left[M_{q}\right]$ and $\boldsymbol{M}^{\prime}=\left[M_{s}^{\prime}\right]$ be two spectra satisfying (a) and (b) above. Then

$$
\tilde{H}_{q+r}\left(M_{q} ; M^{\prime}\right)=\lim _{s \rightarrow \infty} \pi_{q+r+s}\left(M_{q} \wedge M_{s}^{\prime}\right) .
$$

We define $H_{r}\left(\boldsymbol{M} ; \boldsymbol{M}^{\prime}\right)=\lim _{q \rightarrow \infty} \tilde{H}_{q+r}\left(M_{q} ; \boldsymbol{M}^{\prime}\right)$, so that 


$$
H_{r}\left(M ; M^{\prime}\right)=\lim _{q \rightarrow \infty} \lim _{s \rightarrow \infty} \pi_{q+r+s}\left(M_{q} \wedge M_{s}^{\prime}\right) .
$$

Taking the limit in the other order and twisting the factors, we obtain $H_{r}\left(\boldsymbol{M}^{\prime} ; \boldsymbol{M}\right)$, proving

Lemma (2.1). $H_{r}\left(\boldsymbol{M} ; \boldsymbol{M}^{\prime}\right) \approx H_{r}\left(\boldsymbol{M}^{\prime}, \boldsymbol{M}\right)$.

Lemma. (2.2). If $r<q-1$, then

$$
\tilde{H}_{q+r}\left(M_{q} ; M^{\prime}\right) \approx H_{r}\left(M ; M^{\prime}\right) .
$$

Proof. It suffices to show that $\tilde{H}_{q+r}\left(M_{q} ; M^{\prime}\right) \rightarrow \widetilde{H}_{q+r+1}\left(M_{q+1} ; M^{\prime}\right)$ is an isomorphism for $r<q-1$, and for this we must show that

$$
\tilde{H}_{q+r+1}\left(S M_{q} ; M^{\prime}\right) \rightarrow \widetilde{H}_{q+r+1}\left(M_{q+1} ; M^{\prime}\right)
$$

is an isomorphism for $r<q-1$. Regard $S M_{q} \rightarrow M_{q+1}$ as an inclusion, and put $N_{q+1}=M_{q+1} / S M_{q}$. Since $M$ satisfies (a), all three spaces $S M_{q}, M_{q+1}, N_{q+1}$ are $q$-connected. It follows from the generalized suspension theorem [3] that for any of these spaces $\pi_{i}(\quad)=\pi_{i}^{s}(\quad)$ if $i \leqq 2 q\left(\pi_{i}^{s}(\quad)=\tilde{H}_{i}(; S)\right.$ is stable homotopy, where $\boldsymbol{S}$ denotes the sphere spectrum). Since $\boldsymbol{M}$ satisfies (b), the exact sequence for stable homotopy implies that $\pi_{i}^{s}\left(N_{q+1}\right)=0$ if $i \leqq 2 q$. Hence also $\pi_{i}\left(N_{q+1}\right)=0$ for $i \leqq 2 q$, so $N_{q+1}$ is $2 q$-connected. Then the spectral sequence for $\tilde{H}_{*}\left(N_{q+1} ; M^{\prime}\right)$ shows that $\tilde{H}_{i}\left(N_{q+1} ; M^{\prime}\right)=0$ if $i \leqq 2 q$, since $\boldsymbol{M}^{\prime}$ satisfies (a). From the exact sequence for $\widetilde{H}_{*}\left(; \boldsymbol{M}^{\prime}\right)$, it follows that $\widetilde{H}_{i}\left(S M_{q} ; M^{\prime}\right) \rightarrow \widetilde{H}_{i}\left(M_{q+1} ; M^{\prime}\right)$ is an isomorphism if $i<2 q$ (and onto if $i=2 q$ ).

The spectra mentioned at the beginning of this section are multiplicative; this is well known for $K(\pi)$ and $M U$, and has been shown for $b u$ by $\mathrm{D}$. Anderson [2]. The coefficient ring $\widetilde{H}_{*}\left(S^{0} ; b u\right)$ is a polynomial ring over the integers on a 2-dimensional generator, and so has no torsion. The maps $M U(q) \rightarrow B U[2 q]$ mentioned above give rise to a multiplicative map of spectra $v: M U \rightarrow b u([2])$; this induces an epimorphism of coefficient rings $\widetilde{H}_{*}\left(S^{0} ; M U\right) \rightarrow \widetilde{H}_{*}\left(S^{0} ; b u\right)$, which is essentially the Todd genus. Moreover, there are maps $B U[2 q] \rightarrow K(Z, 2 q)$ corresponding to $c_{q, 0} \in H^{2 q}(B U[2 q] ; Z)$, which give rise to another multiplicative map of spectra $\lambda: b u \rightarrow K(Z)$. The composition $M U \rightarrow b u \rightarrow K(Z)$ is the usual map $u: M U \rightarrow K(Z)$ resulting from the cohomology Thom class; this latter map induces the natural transformation $\mu$. Let $\nu$ and $\lambda$ induce $\nu, \lambda$ respectively.

Lemma (2.3). Let $X$ be a $C W$-complex with base point with $\widetilde{H}_{*}(X ; Z)$ free abelian. Then $\widetilde{H}_{*}(X ; M U)$ and $\widetilde{H}_{*}(X ; b u)$ are free abelian and the homomorphisms 


$$
\widetilde{H}_{*}(X ; M U) \stackrel{\nu}{\rightarrow} \tilde{H}_{*}(X ; b u), \widetilde{H}_{*}(X ; b u) \stackrel{\lambda}{\rightarrow} \widetilde{H}_{*}(X ; Z)
$$

are epimorphisms.

Proof. The spectral sequences for $\widetilde{H}_{*}(X ; M U)$ and $\widetilde{H}_{*}(X ; b u)$ both collapse, since $\tilde{H}_{*}(X ; Z)$ and both coefficient rings are free abelian. Thus both $\mu: \widetilde{H}_{*}(X ; M U) \rightarrow \widetilde{H}_{*}(X ; Z)$ and $\lambda$ map onto $\tilde{H}_{*}(X ; Z)$. Let $\left(c_{i}\right)$ be a homogeneous basis for $\tilde{H}_{*}(X ; Z)$ as a free abelian group. Select a homogeneous pre-image $\gamma_{i}$ of $c_{i}$ in $\widetilde{H}_{*}(X ; M U)$; then the image $\gamma_{i}^{\prime}$ of $\gamma_{i}$ in $\widetilde{H}_{*}(X ; b u)$ is also a pre-image of $c_{i}$. It follows easily, from an argument used by $\mathrm{P}$. Conner and E. Floyd [6], that $\widetilde{H}_{*}(X ; M U)$ is a free $\widetilde{H}_{*}\left(S^{0} ; M U\right)$-module on the $\gamma_{i}$, and that $\tilde{H}_{*}(X ; b u)$ is a free $\widetilde{H}_{*}\left(S^{0} ; b u\right)$-module on the $\gamma_{i}^{\prime}$. Thus all assertions are proved.

Q.E.D.

As a consequence of these lemmas, we obtain commutative diagrams such as

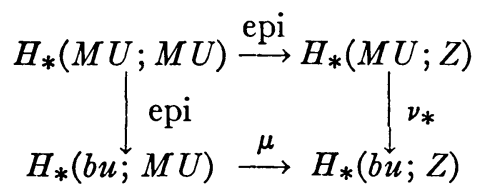

showing that $\operatorname{im}(\mu)=\operatorname{im}\left(\nu_{*}\right)$ in $H_{*}(b u ; Z)$. There is a similar diagram if $b u$ is replaced by $K(\pi), \pi$ cyclic, and also diagrams such as

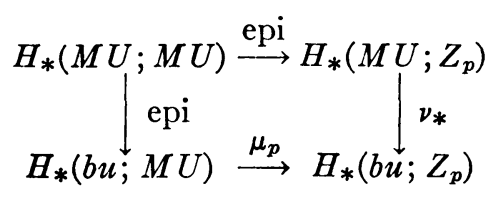

showing that $\operatorname{im}\left(\mu_{p}\right)=\operatorname{im}\left(\nu_{*}\right)$ in $H_{*}\left(b u ; Z_{p}\right)$.

3. Proof of Theorem (1.2). To begin, we remark that for any of the spaces $K(\pi, q), \pi$ cyclic of prime or infinite order, or $B U[2 q]$, the cohomology in the stable range has no elements of order $p^{2}$ for any prime $p$. This is shown by Adams [1] in the latter case, and is well known in the other cases. By the universal coefficient theorem the stable homology of these spaces has no elements of order $p^{2}$. Since the homology groups are finitely generated, Lemma (1.1) follows immediately.

Theorem (1.2) is immediate for $K(\pi, q)$ if $\pi$ is a finite cyclic group of prime order. With $\pi=Z$, the Hurewicz theorem implies that, in dimension $q, \mu$ and all $\mu_{p}$ are onto. Thus we confine attention to dimensions $>q$. Let $x \in \widetilde{H}_{q+r}(K(Z, q) ; Z)$ with $\rho_{p}(x)=\mu_{p}\left(y_{p}\right)$ for all primes $p$. Since $\widetilde{H}_{q+r}\left(K(Z, q) ; Z_{p}\right)=0$ for almost all primes $p$, we may 
assume that almost all the $y_{p}$ vanish. By elementary number theory, if we replace $y_{p}$ by a suitable multiple we may also assume that $\mu_{p^{\prime}}\left(y_{p}\right)=0$ if $p^{\prime} \neq p$. Then putting $y=\sum y_{p}$, we have $\rho_{p}(x)=\mu_{p}(y)$ for all, $p$, i.e., $\rho_{p}(x-\mu(y))=0$ for all $p$, so by Lemma (1.1) $x=\mu(y)$. Thus Theorem (1.2) is proved in this case.

Finally we consider $B U[2 q]$, first proving Proposition (1.5). Recall that the classes $\operatorname{ch}_{q, r} \in H^{2 q+2 r}(B U[2 q] ; Z)$ defined by Adams [1] pass in rational cohomology to $m(r) \operatorname{ch}_{q+r}$, where

$$
m(r)=\prod_{p} p^{[r /(p-1)]}
$$

and $\mathrm{ch}_{q+r}$ is the component of the Chern character in dimension $2 q+2 r$. We wish to construct a class $y_{q, r} \in \tilde{\mathcal{U}}_{2 q+2 r}(B U[2 q])$ so that $\left\langle\mu\left(y_{q, r}\right), \mathrm{ch}_{q, r}\right\rangle=1$, provided that $r<q$. That is, we seek a $u$-manifold $M^{2 q+2 r}$ and $\alpha \in K(M)$ so that $[M]=0$ in the complex bordism ring and $\alpha$ is trivial on the $(2 q-1)$-skeleton of $M$, for which $\left\langle m(r) \operatorname{ch}_{q+r}(\alpha)\right.$, $M\rangle=1$.

Let $\omega=\left(i_{1}, \cdots, i_{k}\right)$ be a partition of degree $r$, so that $k \leqq r<q$. Put $M_{\omega}=C P\left(i_{1}+1\right) \times \cdots \times C P\left(i_{k}+1\right) \times S^{2 q-2 k}$ and let $\alpha_{\omega} \in K\left(M_{\omega}\right)$ be the product of the bundles $\eta-1$ on the projective spaces $(\eta$ the Hopf bundle) and the generating bundle of $\tilde{K}\left(S^{2 q-2 k}\right)$. Then $M_{\omega}$ is a $U$-manifold of dimension $2 q+2 r,\left[M_{\omega}\right]=0$ in the complex bordism ring, and $\alpha_{\omega}$ is trivial on the $(2 q-1)$-skeleton of $M_{\omega}$. Thus a classifying map for $\alpha_{\omega}$ lifts to a map $M_{\omega} \rightarrow B U[2 q]$, and so we obtain $y_{\omega} \in \tilde{u}_{2 q+2 r}(B U[2 q])$. Put $(\omega+1) !=\left(i_{1}+1\right) ! \cdots\left(i_{k}+1\right) ! ;$ it is easily seen that

$$
\left\langle\mu\left(y_{\omega}\right), \operatorname{ch}_{q, r}\right\rangle=m(r) /(\omega+1) !
$$

Lemma (3.1). The integers $m(r) /(\omega+1)$ !, as $\omega$ runs through the partitions of degree $r$, have greatest common divisor 1 .

Proof. It suffices to exhibit for each prime $p<r$ a partition $\omega$ of degree $r$ with $m(r) /(\omega+1)$ ! prime to $p$, since $m(r)$ is divisible by no greater primes. Given $p$, let $\omega$ be any partition of degree $r$ with $p-1$ occurring exactly $[r /(p-1)]$ times. Then $p[r /(p-1)]$ divides $(\omega+1)$ !, and so $m(r) /(\omega+1)$ ! is prime to $p$.

Q.E.D.

Proof of (1.5). For each partition $\omega$ of degree $r$, we have constructed $y_{\omega}$ in $\tilde{u}_{2 q+2 r}(B U[2 q])$ with $\left\langle\mu\left(y_{\omega}\right), \operatorname{ch}_{q, r}\right\rangle=m(r) /(\omega+1)$ !. According to the lemma just established, there is an integral linear combination $y_{q, r}=\sum a_{\omega} y_{\omega}$ with $\left\langle\mu\left(y_{q, r}\right), \operatorname{ch}_{q, r}\right\rangle=1$.

Q.E.D.

We now prove Theorem (1.2) for $B U[2 q]$. Concerning the cohomology of $B U[2 q]$, the following remarks are needed (see [1]). For $r$ odd, $r<2 q-2, H^{2 q+r}\left(B U[2 q] ; Z_{p}\right)=0$ for almost all primes; for 
$r<q, H^{2 q+2 r}\left(B U[2 q] ; Z_{p}\right) \approx Z_{p}$ with generator $\rho_{p}\left(\operatorname{ch}_{q, r}\right)$ for almost all primes $p$. Now let $x \in \widetilde{H}_{*}(B U[2 q] ; Z)$ be given in the stable range with $\rho_{p}(x) \in \operatorname{im}\left(\mu_{p}\right)$ for all primes $p$. If $x$ has odd degree, we may argue as above that $x \in \operatorname{im}(\mu)$. Thus suppose $x \in H_{2 q+2 r}(B U[2 q] ; Z)$; by virtue of Proposition (1.5), we may also assume that $\left\langle x, \operatorname{ch}_{q, r}\right\rangle=0$. Hence $\rho_{p}(x)=0$ for almost all primes $p$. Now say $\rho_{p}(x)=\mu_{p}\left(y_{p}\right)$ for all primes $p$, with almost all the $y_{p}$ zero. Again by Proposition (1.5), we may assume that $\left\langle\mu\left(y_{p}\right), \operatorname{ch}_{q, r}\right\rangle=0$ for all $p$, so that $\mu_{p^{\prime}}\left(y_{p}\right)=0$ for almost all primes $p^{\prime}$. Replacing the $y_{p}$ by suitable multiples, we may further require that $\mu_{p^{\prime}}\left(y_{p}\right)=0$ for all prime $p, p^{\prime}\left(p \neq p^{\prime}\right)$. Putting $y=\sum y_{p}$, we see that $\rho_{p}(x)=\mu_{p}(y)$ for all primes $p$, so that $x=\mu(y)$ by Lemma (1.1). This completes the proof.

4. Proof of Theorem (1.4). We begin by proving Lemma (1.3). Recall that the mod $p$ Steenrod algebra $A^{*}(p)$ acts on $\widetilde{H}_{*}\left(X ; Z_{p}\right)$ via the Kronecker index. The two-sided ideal $I^{*}(p)$ in $A^{*}(p)$ generated by the Bockstein $Q_{0}^{(p)}$ is also the left (or right) ideal generated by the elements of odd degree, according to Milnor [10].

Proof of (1.3). In view of the above remark, it suffices to show that any stable operation $\tilde{\mathcal{U}}_{*}(\quad) \rightarrow \widetilde{H}_{*}\left(\quad ; Z_{p}\right)$ of odd degree is trivial. By Alexander-Spanier duality [13], such an operation corresponds to a stable operation $\tilde{\mathcal{U}}^{*}() \rightarrow \tilde{H}^{*}\left(; Z_{p}\right)$, and so to an element of $\widetilde{H}^{*}\left(M U ; Z_{p}\right)$. Since the stable cohomology of $M U$ vanishes in odd dimensions, the result follows.

Q.E.D.

Theorem (1.4) will now be proved for $B U[2 q]$; a simplified, argument suffices to treat $K(\pi, q), \pi$ cyclic of prime or infinite order. It follows from (2.5) that in the stable range $\operatorname{im}\left(\mu_{p}\right)=\operatorname{im}\left(\nu_{*}\right)$ in $\widetilde{H}_{*}\left(B U[2 q] ; Z_{p}\right)$, where $\nu_{*}: \widetilde{H}_{*}\left(M U(q) ; Z_{p}\right) \rightarrow \widetilde{H}_{*}\left(B U[2 q] ; Z_{p}\right)$ is induced by the map $\nu: M U(q) \rightarrow B U[2 q]$. There is also an induced map in cohomology $\nu^{*}: \widetilde{H}^{*}\left(B U[2 q] ; Z_{p}\right) \rightarrow \widetilde{H}^{*}\left(M U(q) ; Z_{p}\right)$. Making use of the Kronecker index, it suffices to show that in the stable range

$$
\operatorname{ker}\left(\nu^{*}\right)=I^{*}(p) \cdot \widetilde{H}^{*}\left(B U[2 q] ; Z_{p}\right) .
$$

According to Adams $[1], \widetilde{H}^{*}\left(B U[2 q] ; Z_{p}\right)$ is isomorphic in the stable range to a direct sum of $A^{*}(p)$-modules isomorphic to $A^{*}(p) / J^{*}(p)$ on generators $\rho_{p}\left(\operatorname{ch}_{q, r}\right)$ with $r=0,1, \cdots, p-2$; here $J^{*}(p)$ is the left ideal generated by $Q_{0}^{(p)} \in A^{1}(p)$ and $Q_{1}^{(p)} \in A^{2 p-1}(p)$. Milnor [10] has shown that $\widetilde{H}^{*}\left(M U(q) ; Z_{p}\right)$ is isomorphic in the stable range to a direct sum of free modules over $A^{*}(p) / I^{*}(p)$, on generators $s_{\omega}$ of degree $2 \mathrm{deg} \omega$, where $\omega$ is any partition containing no integer of the form $p^{i}-1$. Thus elements of $\widetilde{H}^{*}\left(M U(q) ; Z_{p}\right)$ of degree $\leqq 2 q+2(p-2)$ are independent over $A^{*}(p) / I^{*}(p)$ if they are independent over $Z_{p}$. 
Let $\phi: H^{*}(B U(q)) \rightarrow \widetilde{H}^{*}(M U(q))$ denote the Thom isomorphism, and let $\zeta_{q}$ be the universal bundle over $B U(q)$. If $u_{q}$ denotes the $K$-theory Thom class of $\zeta_{q}$, then $\nu^{*}\left(\operatorname{ch}_{q, r}\right)=\operatorname{ch}_{q, r}\left(u_{q}\right)=m(r) \operatorname{ch}_{q+r}\left(u_{q}\right)$; we regard $\tilde{H}^{*}(M U(q) ; Z) \subset \tilde{H}^{*}(M U(q) ; Q)$ as usual. Now $\operatorname{ch}_{q+r}\left(u_{q}\right)$ $=\phi\left(T_{r}\left(-\zeta_{q}\right)\right)$ according to Bott [5], where $T_{r}\left(-\zeta_{q}\right)$ is the component of degree $2 r$ of the Todd class of the "negative" of the bundle $\zeta_{q}$. Therefore $\nu^{*}\left(\mathrm{ch}_{q+r}\right)=\phi\left(m(r) T_{r}\left(-\zeta_{q}\right)\right)$. It has been shown by M. Atiyah and F. Hirzebruch [4] that $m(r) T_{r}\left(-\zeta_{q}\right) \in H^{2 r}(B U(q) ; Z)$ is divisible by no primes, so that for $p$ a prime $\rho_{p}\left[m(r) T_{r}\left(-\zeta_{q}\right)\right] \neq 0$. Hence $\nu^{*}\left[\rho_{p}\left(\operatorname{ch}_{q, r}\right)\right] \neq 0$ for $r=0,1, \cdots, p-2$, so these elements of $\tilde{H}^{*}\left(M U(q) ; Z_{p}\right)$ are independent over $A^{*}(p) / I^{*}(p)$ according to the preceding paragraph. Thus (4.1) is proved, and with it Theorem (1.4) for $B U[2 q]$.

Q.E.D.

REMARK. The image of $\mu_{p}: \mathcal{U}_{*}\left(K\left(Z_{p}\right)\right) \rightarrow H_{*}\left(K\left(Z_{p}\right) ; Z_{p}\right)$ may be best described with the help of the dual Hopf algebra $A_{*}(p)$ to $A^{*}(p)$. Milnor has shown [9] that $A_{*}(p)$ is a tensor product of an exterior subalgebra with a polynomial subalgebra. If we identify $A_{*}(p)$ with $H_{*}\left(K\left(Z_{p}\right) ; Z_{p}\right)$, the image of $\mu_{p}$ is exactly the polynomial subalgebra which is the annihilator of the ideal $I^{*}(p)$ in $A^{*}(p)$.

\section{REFERENCES}

1. J. F. Adams, On Chern characters and the structure of the unitary group, Proc. Cambridge Philos. Soc. 57 (1961), 189-199.

2. D. Anderson, unpublished.

3. M. Atiyah, Bordism and cobordism, Proc. Cambridge Philos. Soc. 57 (1961), 200-208.

4. M. Atiyah and F. Hirzebruch, Cohomologie-Operationen und charakteristische Klassen, Math. Z. 77 (1961), 149-187. 1962.

5. R. Bott, Lectures on $K(X)$, (mimeographed), Harvard Univ., Cambridge, Mass.,

6. P. Conner and E. Floyd, Differentiable periodic maps, Springer, Berlin, 1964.

7. - Torsion in SU-bordism, Mem. Amer. Math. Soc. No. 60 (1966), 74 bb.

8. A. Hattori, Integral characteristic numbers for weakly almost complex manifolds, Topology 5 (1966), 259-280.

9. J. Milnor, The Steenrod algebra and its dual, Ann. of Math. 67 (1958), 150-171.

10. - On the cobardism ring $\Omega^{*}$ and a complex analogue. I, Amer. J. Math. 82 (1960), 505-521.

11. Y. Shikata, On the realizabilities of integral homology classes by orientable submanifolds, Osaka J. Math. 12 (1961), 79-87.

12. R. Thom, Quelques propriêtés globales des variêtés différentiables, Comment. Math. Helv. 28 (1954), 17-86.

13. G. Whitehead, Generalized homology theories, Trans. Amer. Math. Soc. 102 (1962), 227-283.

UNIVERSITY OF VIRGINIA 